\title{
La Cueva de Baltzola (Dima, Bizkaia): un nuevo conjunto de arte parietal paleolítico en un yacimiento clásico*
}

\author{
The Baltzola Cave (Dima, Biscay): a new ensemble of Palaeolithic parietal art from a classic site
}

\author{
Iñaki Intxaurbe ${ }^{a}$, Olivia Rivero ${ }^{b}$, Sergio Salazar $^{b}$ y Diego Garate
}

En memoria de Lydia Zapata, con quien nos hubiera gustado poder compartir este descubrimiento.

\section{RESUMEN}

Este trabajo presenta el descubrimiento y posterior estudio de grafismos rojos de estilo paleolítico en la cueva de Baltzola (Dima, Bizkaia). Las labores de prospección y documentación allí desarrolladas por los arriba firmantes han identificado un modesto conjunto decorado en el que destaca un ideomorfo difícil de caracterizar. Pudiera tratarse de un signo encajable entre los "claviformes cantábricos" (conocidos en cuevas como Altamira, Tebellín o La Pasiega B y C) o "cuadriláteros con apéndice" (identificados en las de La Pasiega A o El Castillo). La rigidez de las tipologías hasta ahora planteadas para clasificar a los ideomorfos, pudiera obviar la existencia de límites muy difusos entre unos y otros. Además es un signo hasta ahora inédito en el oriente de la Cornisa Cantábrica, que permite correlacionar la cavidad de Baltzola con otras situadas en el centro-oeste cantábrico, pero cuya atribución cronológica precisa resulta difícil a falta de dataciones directas.

\begin{abstract}
This work presents the discovery and subsequent study of red marks of Paleolithic style in the cave of Baltzola (Dima, Bizkaia). The authors identify a small set of decorations that include an ideomorph that is difficult to characterize. It may be either "Cantabrian claviform" (present in caves such as Altamira, Tebellin or La Pasiega B and C) or as a "quadrangular sign with appendix" (identified in caves such as La
\end{abstract}

Pasiega A or El Castillo). The rigid typology hitherto used to classify these ideomorphs does not take into account the existence of diffuse limits between them. The Baltzola marking is in any case a sign hitherto unknown in eastern Cantabria Cornice. The cave of Baltzola can be compared others in the west-central Cantabria whose precise chronological attribution is difficult in the absence of direct dating.

Palabras clave: Arte parietal; Paleolítico Superior; Signo; Cornisa Cantábrica; Pintura.

Key words: Parietal Art; Upper Palaeolithic; Sign; Cantabria; Painting.

\section{INTRODUCCIÓN}

El día 25 de septiembre de 2016, en el marco de los cursos de espeleo-socorro organizados por la Euskal Espeleo Laguntza/Espeleo Socorro Vasco, uno de los firmantes (I. I.), identificó unas grafías de posible origen paleolítico que parecían representar una serie de trazos verticales aplicados en rojo. Estaban cerca del acceso principal de la cueva, en el arco de entrada a una galería lateral. El día 14 de marzo de 2017, tres de los firmantes (D. G., O. R. e I. I.), visitaron la cavidad para evaluar dichas grafías, reconociendo un ideomorfo complejo rojo muy próximo a ellas. Tras los trámites administrativos preceptivos realizados con el Servicio de Patrimonio Cultural de la Diputación Foral de Bizkaia, en septiembre y octubre

\footnotetext{
* Este trabajo se enmarca en el proyecto de Investigación "Aprendizaje y desarrollo de las capacidades artísticas en los Humanos Anatómicamente Modernos; un enfoque multidisciplinar" (HAR2017-87739-P) financiado por el Ministerio de Ciencia, Innovación y Universidades, IP: Olivia Rivero. El Servicio de Patrimonio Cultural de la Diputación Foral de Bizkaia financió las investigaciones en la cueva de Baltzola.

a Dpto. Mineralogía y Petrología. Euskal Herriko Unibertsitatea/Universidad del País Vasco. 48940 Leioa, Bizkaia, España. Correo e.: iintxaurbe001@ikasle.ehu.eus https://orcid.org/0000-0003-3643-3177

b Dpto. Prehistoria, Historia Antigua y Arqueología, Universidad de Salamanca. 37002 Salamanca, Salamanca, España.

Correos e.: oliviariver@usal.es https://orcid.org/0000-0002-0730-4854; salazar.laredo@gmail.com https://orcid.org/0000-0002-2892-3945

c Instituto Internacional de investigaciones Prehistóricas de Cantabria (IIIPC), Universidad de Cantabria. 39005 Santander, Cantabria, España. Correo e.: garatemaidagandiego@gmail.com https://orcid.org/0000-0001-6685-9588

Recibido 20-IX-2018; aceptado 19-III-2019
} 
de 2017 se abordó la prospección y el estudio completo del arte parietal de la cueva de Baltzola por parte de los autores de este artículo, contando con la asistencia del Grupo Espeleológico de Matiena (GEMA).

\section{LOCALIZACIÓN Y DESCRIPCIÓN DE LA CAVIDAD}

La cueva de Baltzola se localiza en el promontorio de Kobagan en la ladera sur del monte Bargondia, sobre el pequeño valle cerrado conformado por los altos de Bargondia, Garaio y Basabil (Fig. 1b). El paisaje se abre hacia la cuenca del arroyo de Indusi que desemboca posteriormente en el río Arratia. Pertenece al municipio de Dima, situado en el área interior centro-oriental del Territorio Histórico de Bizkaia. La cueva se asienta a $370 \mathrm{~m} \mathrm{s.} \mathrm{n.} \mathrm{m.,} \mathrm{en} \mathrm{calizas} \mathrm{del} \mathrm{Cre-}$ tácico Inferior de edad Aptiense-Albiense, dentro del karst de Indusi perteneciente al flanco sur del sinclinorio de Bizkaia.

Se accede a la cavidad a través de tres entradas que desembocan en un vestíbulo de unos $150 \mathrm{~m}$ de longitud, que se abre mediante un gran portalón de aproximadamente $50 \times 25 \mathrm{~m}$, orientado al Sur-Sureste. Dos de los accesos principales, Gibeldar y Erdikoate, se orientan al Suroeste en perpendicular al vestíbulo del que salen varias galerías laterales conformando un desarrollo laberíntico de unos $2.160 \mathrm{~m}$ con varios pisos sucesivos (GEMA 1999).

\section{LOS ESTUDIOS ARQUEOLÓGICOS PREVIOS}

La existencia de la cavidad y de su depósito ya es señalada durante el siglo XIX, siendo Baltzola el primer yacimiento arqueológico en cueva de Bizkaia conocido por la bibliografia. Según G. Mortillet (1883) en 1866 el alemán F. Jagor hizo la primera intervención arqueológica, atribuyendo al Magdaleniense los materiales recuperados. Sin embargo, apenas han trascendido los detalles de esta actuación. En 1912, A. Gálvez Cañero (1912) excava Baltzola entre otras cavidades vizcaínas. Su información, también imprecisa, señala la recogida de industria del Magdaleniense y Aziliense en sílex en los taludes cercanos a la boca, comparándola con la localizada por F. Jagor. En cambio considera de aspecto Neolítico otra procedente de una galería superior. Se desconoce el paradero de los materiales de ambas intervenciones.

J. M. Barandiarán (1932) hace un nuevo sondeo en el yacimiento de Baltzola sobre el que tampoco se aporta información precisa. La indefinición del croquis
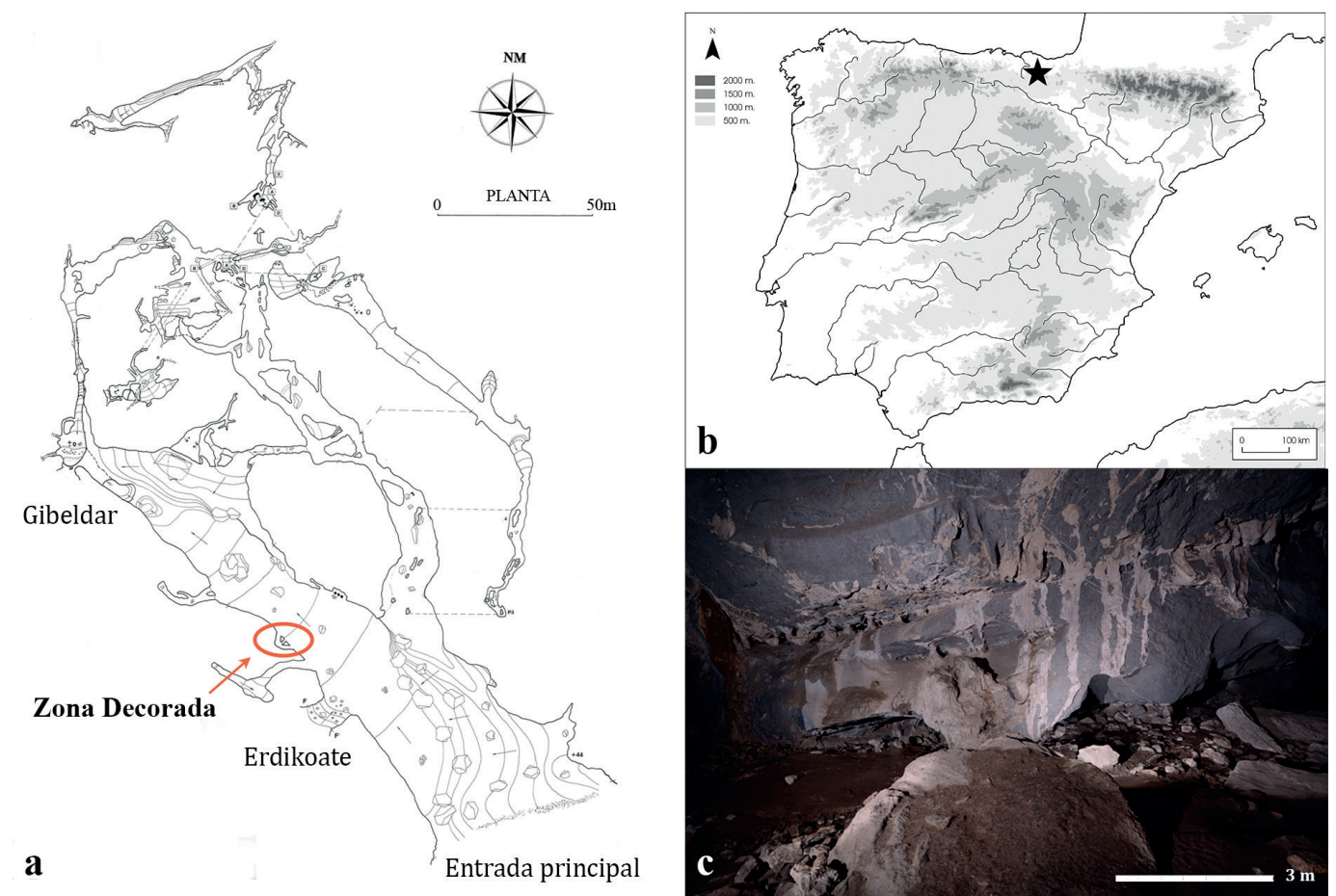

Fig. 1. Cueva de Baltzola (Dima, Bizkaia): a. plano de la cavidad con indicación del sector decorado (topografía del grupo espeleológico GEMA de Matiena, 1994); b. ubicación de la cueva de Baltzola en la península ibérica; c. fotografía del sector donde se ubican las unidades gráficas identificadas.

Trab. Prehist., 76, N. ${ }^{\circ}$ 2, julio-diciembre 2019, pp. 323-334, ISSN: 0082-5638

https://doi.org/10.3989/tp.2019.12240 
hace difícil localizar la excavación en la cavidad. Los materiales -núcleos microlaminares, raspadores, buriles, laminillas, retocadores, etc.- aparecen hasta a 30 $\mathrm{cm}$ de profundidad y son atribuidos al final de Tardiglaciar. Además se recuperan fragmentos cerámicos sin decoración en superficie. La información aportada por J. M. Barandiarán permite, al menos, tener una idea aproximada de la estratigrafía, algo que no ocurre en el caso de las intervenciones arqueológicas previas.

La contribución de E. Berganza Gochi et al. (1979) al yacimiento de Baltzola es diferente. Excavan un enterramiento colectivo en una galería colgada en el lateral derecho del portalón de la cueva que atribuyen al Calcolítico y a la Edad de Bronce en función del ajuar recuperado.

En 2006 L. Zapata Peña dirigió un nuevo proyecto arqueológico con el fin de conocer las secuencias crono-cultural del yacimiento y paleoambiental del entorno de Axlor y Baltzola, así como de dar continuidad a las actividades arqueológicas previas. Tras dos campañas de sondeos (2006, 2007), se desarrolló un proyecto cuatrienal de excavación de Baltzola desde 2008 hasta 2012 (Zapata et al. 2007, 2008, 2009; San Pedro et al. 2010; Zapata et al. 2011; Regalado et al. 2012, 2014). Lamentablemente el fallecimiento de la directora truncó el proyecto.

En el vestíbulo principal se han excavado dos cuadros de $1 \mathrm{~m}^{2}$ cada uno. La estratigrafía (algo más de $1,5 \mathrm{~m}$ de potencia) tiene nueve niveles con una secuencia de Prehistoria reciente con cerámica (Zapata et al. 2009). En la repisa superior derecha, también conocida como el redil, se han abierto cuatro cuadros completos y dos mitades de cuadro hasta 2009. En uno se alcanzó la base, en una zona que podría corresponder a la de intervención de J. M. Barandiarán en 1932. En 2010 se excava otro cuadro junto a la pared oeste del vestíbulo, con similitudes estratigráficas con los niveles del redil (Regalado et al. 2012). Se han diferenciado doce unidades estratigráficas numeradas por orden de aparición durante la excavación. La información disponible es escasa por la interrupción del proyecto de investigación, pero la mayoría de los niveles cuentan con asignación crono-cultural de (San Pedro et al. 2010):

Nivel 12: arqueológicamente estéril en su reducida superficie.

Nivel 11: excavado en una superficie muy escasa. Los materiales en sílex y en lutita sugieren una cronología musteriense, mientras que la fauna presenta restos de cabra montés y de gran bóvido.

Nivel 10: posible magdaleniense.

Nivel 8: una datación sobre escápula de ciervo lo sitúa en el Magdaleniense Superior Final (12.160 \pm 60 BP).

Nivel 7: abundante ciervo. Una datación sobre hueso atribuye el nivel al Magdaleniense Superior Final $(12.440 \pm 60 \mathrm{BP})$.
Nivel 6: abundantes restos de industria y fauna entre la que destaca el ciervo.

Nivel 9: a techo presenta fragmentos de cerámica que indican una formación post- paleolítica, pero la base se caracteriza por piezas líticas de dorso. Además, un fragmento óseo con decoración incisa oblicua y "escaleriformes", datado en 10.270 \pm 60 BP (Zapata et al. 2009), indica una cronología aziliense.

El descubrimiento de las manifestaciones de arte parietal paleolítico precisó del diseño de un proyecto de prospección y documentación general de la actividad gráfica presente en la cavidad.

\section{METODOLOGÍA DE ESTUDIO DEL ARTE PARIETAL}

\subsection{Prospección}

La prospección sistemática de las paredes y de los techos consistió en un reconocimiento detenido de todas las superficies rocosas accesibles directa o indirectamente. El grupo de espeleología GEMA supervisó el material y la técnica de progresión espeleológica precisadas, acompañándonos en las labores de prospección realizadas en el interior de la cueva. El mal estado de conservación de la cavidad, repleta de grafitis modernos (en gran parte grabados mediante instrumentos metálicos) que jalonan la mayoría de sus paredes condicionó en todo momento la prospección. También son numerosos los desperdicios dejados por los visitantes a lo largo de las galerías, ya que la cueva es de fácil acceso y muy visitada.

\subsection{Documentación arqueométrica}

La documentación escrita y la catalogación se hicieron in situ tras la prospección. Se empleó la observación visual con lupas de aumento (microscopio digital Dinolite) y fichas estándar en las que se recogió información general y específica. Se establecieron tres crecientes de registro para el conjunto del repertorio gráfico parietal. El de grafía o unidad gráfica para los motivos individualizados; el de panel o unidad compositiva para contextualizar los motivos dentro de cada uno de los lienzos decorados y el de sector o unidad topográfica para referirse al correspondiente sector decorado.

\subsection{Documentación fotográfica y restitución infográfica de los motivos}

La documentación se basó en la fotografía digital convencional y la fotogrametría para obtener modelos

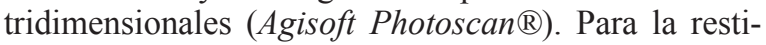


tución gráfica de las imágenes hemos recurrido al tratamiento digital (plug-in D-stretch para ImageJ®) (Le Quellec et al. 2015) y a la infografía (paquetes informáticos de Adobe $(\mathrm{C})$. Además de la materia colorante hemos creído imprescindible incluir las características del soporte y la escala de la restitución. Las correcciones pertinentes se realizaron directamente in situ en la versión digital.

\section{DESCRIPCIÓN DEL DISPOSITIVO GRÁFICO PARIETAL}

La prospección sistemática de la cavidad ha permitido documentar un único sector decorado (sector A) ubicado a poca distancia del área vestibular, frente a la excavación arqueológica. Esta zona se halla en la galería principal de la cueva de Baltzola, cerca de su entrada principal, a unos $20 \mathrm{~m}$ de la boca de Erdikoate (Fig. 1a). En la pared izquierda de esta galería, en dirección a la boca de Gibeldar, se abre un pequeño divertículo con 3 entradas. En este sector las grafías se encuentran en dos paneles, situados junto a la primera y segunda entrada (Fig 1c).

\subsection{PANEL I}

El panel I está bajo la primera boca de entrada al sector. Esta tiene forma de un arco largo de $3 \mathrm{~m}$ de anchura. La altura (1 m respecto al suelo actual) obliga a permanecer en postura sentada o tumbada para observar la figura, situada a $99 \mathrm{~cm}$ del suelo actual. Desconocemos la altura del suelo en el momento de la ejecución de la Grafía I.1, el único motivo que contiene. Consiste en una serie de al menos siete concentraciones de pigmento rojo descolorido de tendencia vertical y una horizontal que presumiblemente han sido trazadas con un lápiz en seco, atendiendo a la finura del trazo y a su interrupción con los accidentes del soporte (Fig. 2c). La pared es una superficie cóncava horizontal de caliza, con formaciones calcíticas de tipo coliflor y algunos desconches que no afectan a la grafía. La primera concentración y la última están embe-
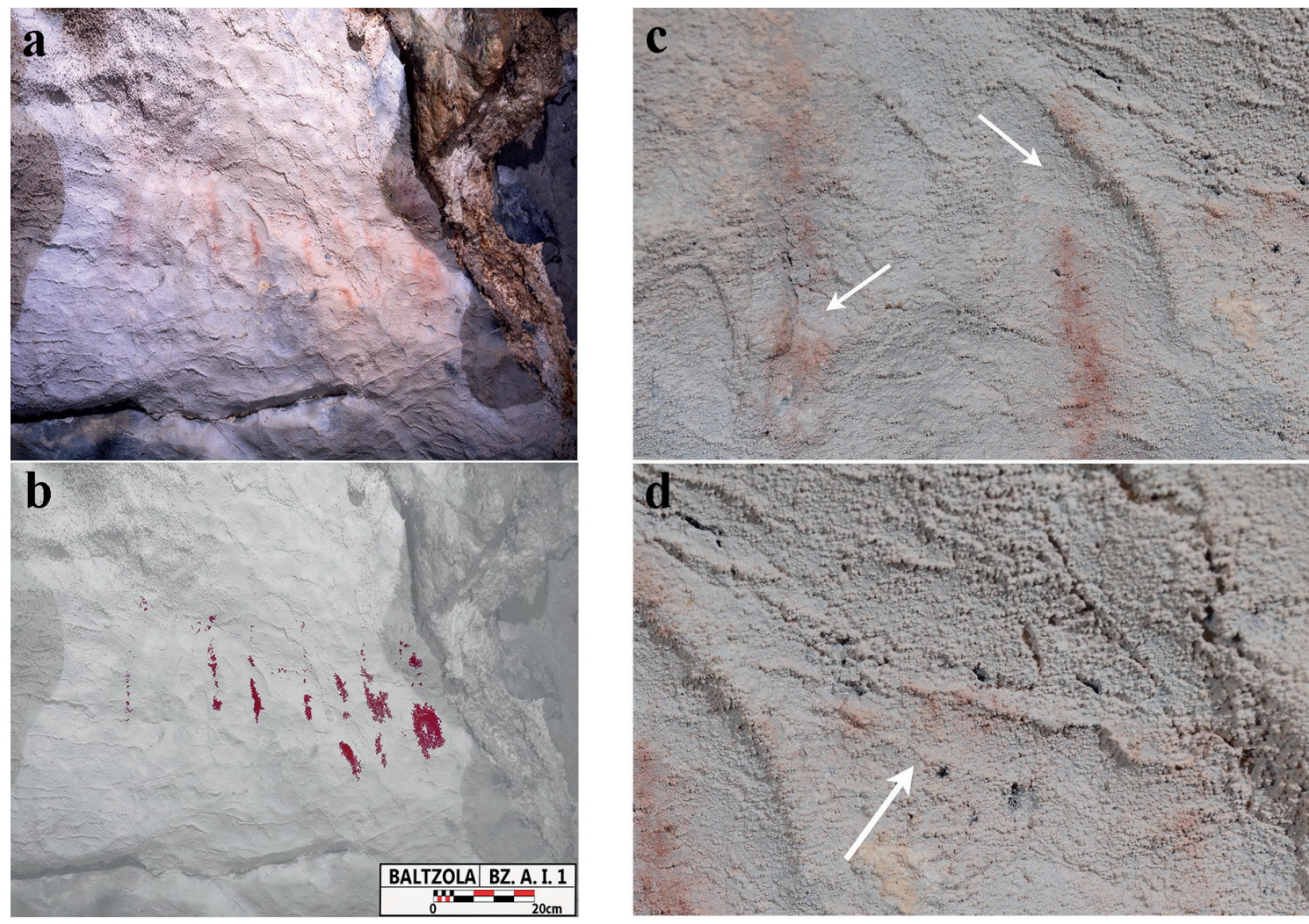

Fig. 2. Grafía I.1. del Panel I del sector decorado de la cueva de Baltzola: a-b. fotografía y calco (la restitución gráfica ha sido realizada empleando paquetes informáticos de Adobe (C); para la saturación de color se ha empleado el plug-in D-stretch para ImageJ®); c. detalles de la interrupción del trazo con los accidentes del soporte; d. restos de una posible línea horizontal (en color en la edición electrónica).

Trab. Prehist., 76, N. ${ }^{\circ}$ 2, julio-diciembre 2019, pp. 323-334, ISSN: 0082-5638

https://doi.org/10.3989/tp.2019.12240 
bidas por las concreciones. Las líneas son de tendencia rectilínea, menos la última, cuyo desarrollo es más extendido. El tercer trazo de la serie empezando por la izquierda, la mejor conservada, contiene un abultamiento lateral derecho. Los trazos se extienden por una superficie con una media de $28 \mathrm{~cm}$ de altura y 58 $\mathrm{cm}$ de anchura máxima (Fig. 2a,b). Este motivo presenta un tamaño similar al de la segunda figura identificada sobre cuya identificación como signo convencional de tradición cantábrica no tenemos dudas. Teniendo esto en cuenta podríamos barajar la categorización de esta primera unidad gráfica como otro ideomorfo, un cuadrilátero de lados mayores curvos y relleno de trazos verticales, p. ej. Sin embargo los vestigios muy difusos de pigmento en la zona de la línea horizontal (Fig. 2c,d) que parecen una migración del pigmento y el que las líneas verticales se prolonguen por encima de él hacen más plausible su identificación como agrupación de trazos de tendencia vertical. Signos similares a la Grafía I.1 de Baltzola se observan en otros conjuntos cantábricos como Altamira (Cartailhac y Breuil 1906; Breuil y Obermaier 1935) o la zona IV de La Garma (González Sainz 2003).

\subsection{Panel II}

El panel II se encuentra en la pared derecha de la galería a la que da acceso la segunda boca de entrada al divertículo y a $2 \mathrm{~m}$ de la entrada que mide $130 \mathrm{~cm}$ de anchura por $175 \mathrm{~cm}$ de altura. El panel es observable desde la galería principal de la cueva.

La Grafía II.1., la única existente, se sitúa en un punto de la galería que mide $180 \mathrm{~cm}$ de altura por el que actualmente podemos circular en posición erguida. Se trata de un signo complejo en rojo con base muy ancha y dos apéndices triangulares en su parte central, en direcciones opuestas. Además, parece poseer dos líneas paralelas exteriores a la base central de la figura, interrumpidas por los citados apéndices. Pudiera representar a dos "claviformes cantábricos" yuxtapuestos, quizás compartiendo una misma base, o a un "cuadrilátero acolado" con dos apuntamientos. El estado de conservación actual impide decantarnos por una opción u otra (Fig. 3). Está trazado sobre una superficie de textura lisa de tendencia plana/cóncava. El colorante está embebido en la propia caliza, salvo en su parte derecha donde el pigmento se mezcla con una colada de calcita.

El signo mide $47 \mathrm{~cm}$ de longitud y $27 \mathrm{~cm}$ de anchura media en la base. La anchura máxima está en su zona central donde se ubicaría(n) el(los) apéndice(s) del mismo. Se alza a $142 \mathrm{~cm}$ del suelo actual, que pudiera corresponder al que había en el momento de ejecución de la grafía, ya que la altura es óptima para su realización y visualización.
El sector decorado presenta una tercera boca de entrada que mide $4 \mathrm{~m}$ por $2 \mathrm{~m}$ de altura, desde la que, tras un recorrido de $8 \mathrm{~m}$ como máximo, se llega al panel I. Además de los paneles I y II, no hemos localizado ninguna evidencia arqueológica en este sector, cuya cercanía a la boca principal lo ha anegado bastante de basuras recientes y material orgánico en descomposición. Esto dificulta incluso la identificación de grafitis en las paredes o techos de la cueva.

La distribución espacial, la homogeneidad cromática y el similar tamaño de los motivos, apuntan hacia una probable sincronía de la totalidad del conjunto pictórico reconocido.
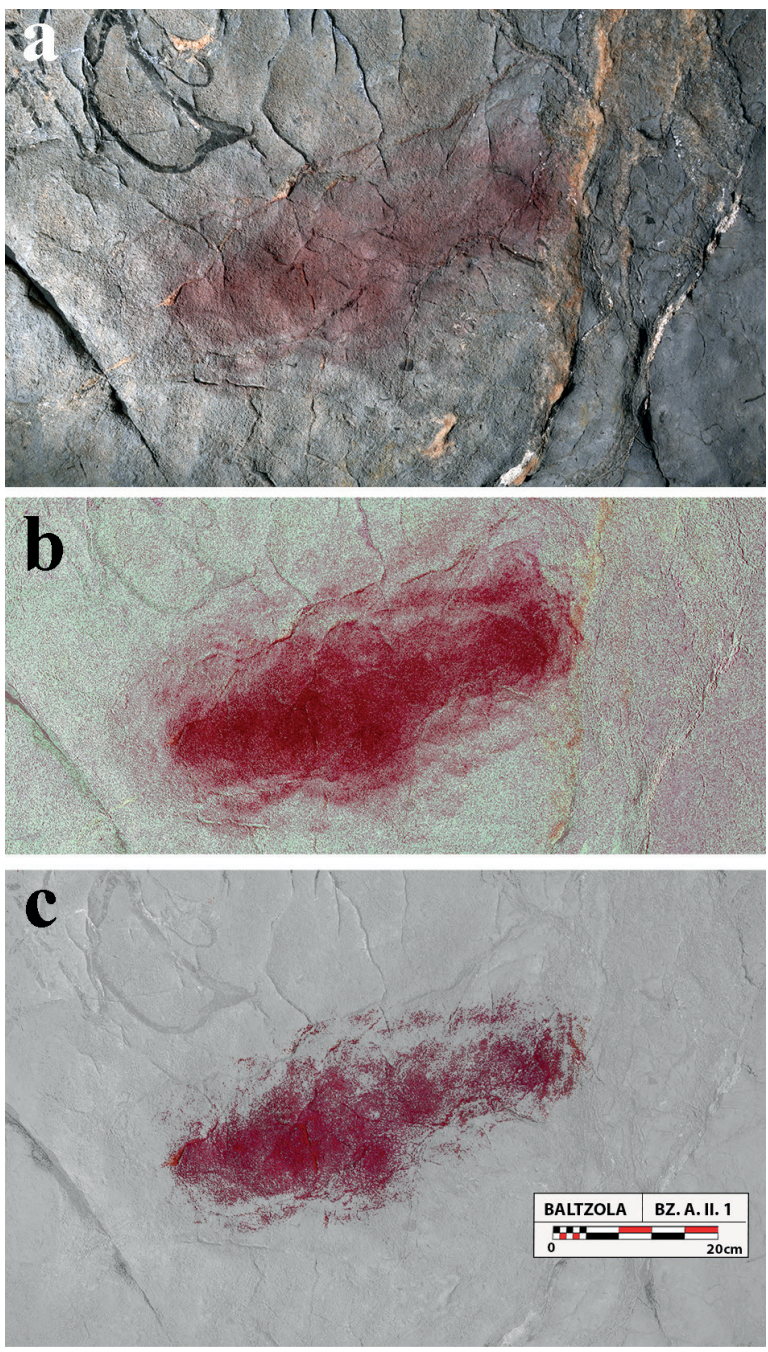

Fig. 3. Grafía II.1. del Panel II del sector decorado de la cueva de Baltzola: a. fotografía de la unidad gráfica; b. saturación de color obtenida mediante el plug-in D-stretch para ImageJ $囚$; c. calco de la unidad gráfica (en color en la edición electrónica). 


\section{EVALUACIÓN}

\subsection{Baltzola en su contexto geográfico local}

El modesto conjunto decorado de Baltzola es el ejemplo más meridional de arte parietal paleolítico encontrado hasta la fecha en el oriente de la Cornisa Cantábrica al situarse en el curso alto de la cuenca del río Arratia (afluente a su vez del río Ibaizabal), muy cerca de la divisoria de aguas cantábrico/ mediterráneas. Es, por lo tanto, una zona de un notable interés estratégico. La presencia de grupos humanos allí está atestiguada desde el Paleolítico Inferior por los restos hallados en la cueva de Arlanpe (Ríos-Garaizar et al. 2013). A ellos se suman ocupaciones musterienses del Paleolítico Medio en Axlor, Askondo y la propia Baltzola. Durante el Paleolítico Superior el registro arqueológico aumenta de manera considerable con ocupaciones más o menos continuadas en las cuevas de Bolinkoba y Baltzola y más puntuales en las de Askondo, Atxuri I, Axlor y Silibranka (Barandiarán 1950, 1961, 1964, 1980; San Pedro et al. 2010; Garate y Ríos-Garaizar 2013).

La identificación de arte parietal es más problemática. En 1960 se detecta una posible grafía en rojo en la cueva de Atxuri I (Fernández García de Diego 1971), pero su posterior destrucción por una cantera y la ausencia de cualquier documento fotográfico o estudio obliga a ser cautos respecto a su aceptación. La presencia de arte parietal paleolítico en el interior oriental de la Cornisa Cantábrica se verifica en 2011, con el descubrimiento de las pinturas rojas gravetienses de la cueva de Askondo (Garate y Ríos-Garaizar 2011, 2012, 2013). Recientemente se ha señalado la existencia de grabados de dudosa factura en la cueva de Bolinkoba (García-Díez 2015). El discreto conjunto rupestre de Baltzola es el tercer conjunto identificado en la zona. Se enmarca en los descubrimientos que se vienen sucediendo en los últimos años y van llenando el mal llamado "vacío vasco" de arte rupestre paleolítico (Garate 2018).

\subsection{En los límites de la tipología: los "claviformes cantábricos" y los "cuadriláteros con apéndice"}

La identificación de un signo complejo entre los motivos de la cueva de Baltzola ha permitido correlacionar este nuevo conjunto con otros situados en el centro/oeste de la Cornisa Cantábrica. No obstante, el estado actual de conservación de la grafía impide discernir si se trata de un "claviforme cantábrico" o de un "cuadrangular acolado".

\subsubsection{Claviformes cantábricos}

La grafía identificada puede ser interpretada como dos "claviformes cantábricos" yuxtapuestos con los apéndices apuntando en direcciones opuestas, similares a los motivos 1a y $1 \mathrm{~b}$ del primer conjunto de la cueva de Tebellín (Llanes, Asturias) (MartínezVilla 2017: 83). Las grafías de Baltzola parecen compartir la misma base, ya que no se distingue la separación entre los motivos del citado conjunto de Tebellín (Fig. 4).

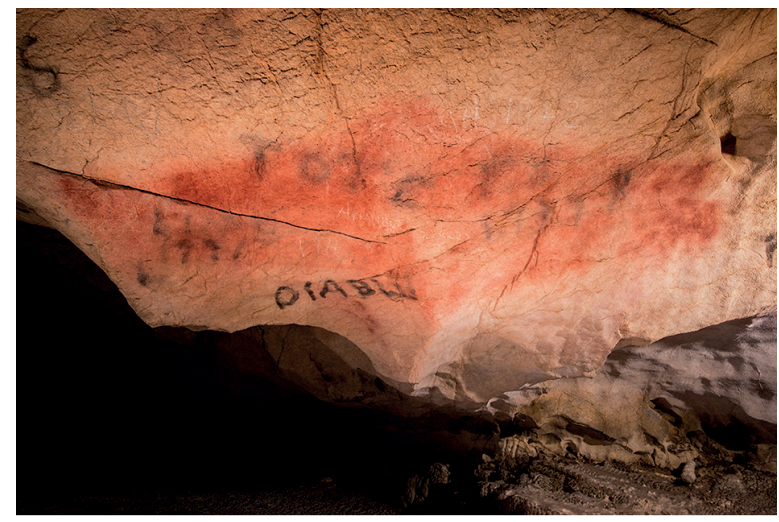

Fig. 4. Motivos 1a y $1 \mathrm{~b}$ de la cueva del Tebellín, Llanes, Asturias (selección de Martínez-Villa 2017: 82, fig. 2c) (en color en la edición electrónica)

La estructura de estos ideomorfos, denominados también "claviformes clásicos" (González Sainz 1993) está formada por una base de tendencia rectangular (ancha o estrecha) con una protuberancia lateral (triangular o rectangular). Algunos tienen los apéndices laterales curvados. Todos los documentados hasta la fecha muestran una simetría vertical y están realizados con pintura roja. Por ello es fácil diferenciarlos de los "claviformes tardíos" o "pirenaicos" presentes en otros contextos cronológicos y geográficos, y con una morfología y técnica más diversas ${ }^{1}$.

Partiendo del análisis publicado por A. MartínezVilla (2017), hemos diferenciado cinco morfotipos entre el repertorio completo de estos signos, repartidos

\footnotetext{
${ }^{1}$ Ideomorfos adscritos culturalmente a momentos del Magdaleniense Medio/Superior y en contextos geográficos pirenaicos mayoritariamente (Tuc d'Audoubert, Trois Frères, Bedeilhac, Niaux, Portel, Fontanet, Mas d'Azil), si bien también hay algunos ejemplos en la región francesa del Lot (Sainte-Eulalie, Mazet) y en la Cornisa Cantábrica (El Pindal, Cullalvera -en soporte parietal y mueble- y Armintxe) (Lorblanchet et al. 1973; Vialou 1986; Raux 1996; González Sainz et al. 1997; Begouen et al. 2009; Díaz y Astorqui 2012; Menéndez 2014; González Sainz 2017).
} 
en 8 cavidades de la Cornisa Cantábrica: La Lloseta y Tito Bustillo (Ribadesella, Asturias) (Balbín 2014); Tebellín (Posada de Llanes, Asturias) (Martínez-Villa 2017); Las Aguas (Novales, Alfoz de Loredo, Cantabria) (Montes Barquín et al. 2016); Altamira (Santillana del Mar, Cantabria) (Breuil y Obermaier 1935; Cartailhac y Breuil 1906); La Pasiega B y C (González Sainz y Balbín 2010) y El Castillo (Puente Viesgo, Cantabria) (Mingo Álvarez 2010) y La Garma (Omoño, Ribamontán al Monte, Cantabria) (González Sainz 2003) (Fig. 5).

Los morfotipos se basan en la morfología de sus bases (ancha o estrecha), la curvatura de sus extremos y las formas de los apéndices (triangulares o rectangulares) (Fig. 6):

- Base estrecha y apéndice rectangular:en los conjuntos de La Pasiega B y C y en el del techo de los polícromos de Altamira.

- Base estrecha y apéndice triangular: Tito Bustillo (posibles), Tebellín, Las Aguas, Altamira y La Pasiega C.

- Base ancha y apéndice triangular: Tebellín, Altamira, El Castillo y La Garma.

- Base ancha y apéndice triangular, con los extremos en curva: Tebellín, Altamira y La Pasiega B.
- Base muy ancha y apéndice triangular: La Lloseta, Tebellín, La Pasiega C, La Garma.

Aquí podría encajar la evidencia parietal de la cueva de Baltzola (dos claviformes yuxtapuestos) que presentamos en este artículo

Los signos de Tebellín y Baltzola conforman el total del corpus del conjunto pictórico junto a otras

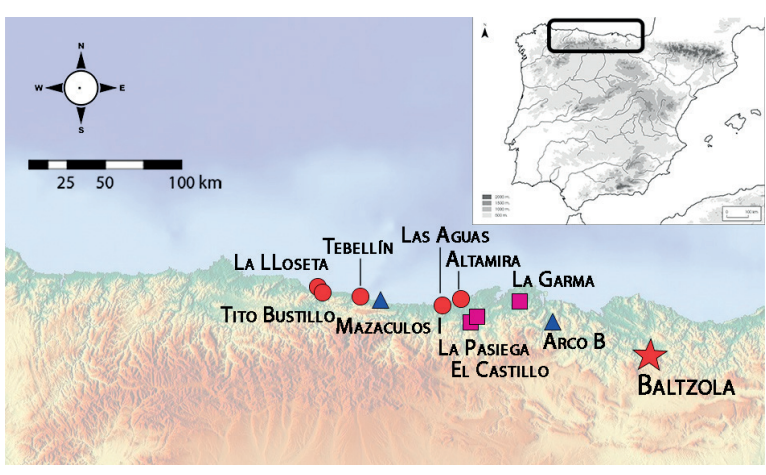

Fig. 5. Conjuntos parietales mencionados en el texto. El círculo localiza los sitios con "claviformes cantábricos", el triángulo aquellos con "cuadriláteros con apéndice" y el cuadrado los que contienen ambos. La estrella sitúa la cueva de Baltzola (en color en la edición electrónica).

\begin{tabular}{|c|c|c|c|c|c|c|}
\hline & $\begin{array}{c}\text { Base estrecha } \\
\text { y apéndice } \\
\text { rectangular }\end{array}$ & $\begin{array}{l}\text { Base estrecha } \\
\text { y apéndice } \\
\text { triangular }\end{array}$ & $\begin{array}{l}\text { Base ancha y } \\
\text { apéndice } \\
\text { triangular }\end{array}$ & $\begin{array}{l}\text { Base ancha y } \\
\text { apéndice } \\
\text { triangular y } \\
\text { extremos en } \\
\text { curva } \\
\end{array}$ & $\begin{array}{l}\text { Base muy } \\
\text { ancha y } \\
\text { apéndice } \\
\text { triangular }\end{array}$ & TOTAL \\
\hline Tito Bustillo & & 3 posibles & & & & ¿3?* \\
\hline La LLoseta & & & & & 1 & 1 \\
\hline Tebellín & & 1 & 4 & 3 & 3 & 11 \\
\hline Las Aguas & & 1 & & & & 1 \\
\hline Altamira & 2 & 14 & 16 & 4 & & 36 \\
\hline La Pasiega B & 3 & & & 7 & 7 & 17 \\
\hline La Pasiega C & 2 & 1 & 2 & & 2 & 7 \\
\hline El Castillo & & & $2(+4$ posibles $)$ & & & $2(+i 4 ?)^{*}$ \\
\hline La Garma & & & 2 & & 1 & 3 \\
\hline Baltzola & & & & & 2 posibles & ¿2?* \\
\hline TOTAL & 7 & $17(+3$ posibles $)$ & $26(+4$ posibles $)$ & 14 & 14 ( +2 posibles) & $78(+i 9 ?)^{*}$ \\
\hline & & & & & & \\
\hline
\end{tabular}

Fig. 6. Repartición por cuevas de los signos claviformes cantábricos en función de los tipos indicados. *Los números situados entre interrogaciones señalan los posibles signos claviformes. 
puntuaciones, líneas y manchas informes. No existe ningún otro ideomorfo complejo ni figura animal. No obstante, sus características estructurales (disposición agrupada) y espaciales (espacios laterales) son idénticas a las de los signos que en otras cavidades sí comparten espacios con registros gráficos mucho más amplios temática, técnica y cronológicamente.

En Tebellín se reconocen claviformes cantábricos pertenecientes a 4 de los 5 morfotipos mencionados en este artículo. Baltzola compartiría con Tebellín el grupo de claviformes cantábricos de base muy ancha y apéndice triangular, si se aceptara nuestra interpretación. Además, el sector decorado de ambas cuevas se reduce a un pequeño desarrollo lateral parcialmente visible desde la estancia principal, donde no se evidencian representaciones parietales.

La cronología atribuida a estos signos ha variado con la historia de la investigación. Se han vinculado al estilo IV de Leroi-Gourhan (1968), en concreto a su fase antigua (González Morales 1982) o se ha planteado su anterioridad a los grabados de "ciervas estriadas" del Magdaleniense Inferior cantábrico (Fortea 2005). Otros investigadores los consideran una evolución de los signos cuadrangulares y ovales con apuntamiento, o de los signos acolados (Leroi-Gourhan 1981), proponiendo incluso las derivaciones específicas presentes en la Cornisa Cantábrica (González Sainz y González Morales 1986) y atribuyéndoles una cronología del Magdaleniense Inferior cantábrico (Balbín y González-Sainz 1994, 1996; González Sainz 2012). Sin fechas absolutas directas, ni paralelos conocidos en el arte mueble, la datación de este tipo de ideomorfos resulta actualmente bastante comprometida.

Se podría añadir, como ya se ha apuntado, que en 7 de los 9 conjuntos conocidos (La Lloseta, Tito Bustillo, Altamira, La Pasiega B y C, El Castillo y La Garma) algunos de estos signos están situados próximos a paneles de figuras animales rojas de cronología gravetiense y solutrense (Garate 2010). En Altamira podemos añadir la datación indirecta de un conjunto de claviformes rojos de base ancha con extremos en curva del techo de polícromos. La técnica del U/Th arrojó un resultado ante quem de $36.160 \pm 610$ años (Pike et al. 2012). Sin embargo, como en la mayoría de los casos mencionados las cavidades tienen una larga tradición gráfica, es complicado relacionar a estas figuras con una fase de decoración concreta. También es difícil ese tipo de conexión en la cueva de Las Aguas, sin alguna figura animal roja asociada. Finalmente, existe una problemática superposición en el techo de polícromos de Altamira que alberga el mayor conjunto de estos signos junto a decoraciones pertenecientes estilísticamente a la práctica totalidad de las culturas reconocidas en el Paleolítico Superior. Uno de estos ideomorfos se superpo- ne probablemente ${ }^{2}$ a la pata delantera de una cierva policromada, adscrita culturalmente a la misma fase de ejecución de los bisontes polícromos y datada radiocarbónicamente en el Magdaleniense Medio. De ser así, podríamos estar hablando de una tradición vigente durante la mayor parte del Paleolítico Superior, o, al menos, del uso de estos signos como tema recurrente a lo largo de las diferentes fases cronoculturales reconocidas.

\subsubsection{Cuadriláteros con apéndice}

El motivo de Baltzola puede interpretarse alternativamente como un signo cuadrangular acolado con la particularidad de contar con dos apéndices, superior e inferior, en vez de uno solo (característica hasta ahora inédita). Carecería de divisiones internas, ya que el interior se presenta relleno de pintura roja. No obstante, cuenta con dos líneas paralelas al borde exterior de la figura, que pudieran integrarse con el resto del motivo.

Los signos rectangulares, cuadriláteros o cuadrangulares de la Cornisa Cantábrica se reparten en 20 conjuntos diferentes ${ }^{3}$, cuya diversidad tipológica, técnica y, seguramente, cronológica supera la de los claviformes. Recientemente, se han caracterizado en función de sus divisiones internas, de la presencia o no de escaleriformes y de la presencia o no de apéndices laterales (Sauvet et al. 2018). Si nos ceñimos a este último grupo, al que pertenecería hipotéticamente el ideomorfo encontrado en Baltzola, sus integrantes se reparten en siete conjuntos de la Cornisa Cantábrica: Mazaculos I (Ribadedeva, Asturias) (Gómez et al. 1991), La Pasiega A, C y D (González Sainz y Balbín 2010), El Castillo (Mingo Álvarez 2010) (Puente Viesgo, Cantabria), La Garma (González Sainz 2003) (Omoño, Ribamontan al Monte, Cantabria) y Arco B (Pondra, Ramales de la Victoria, Cantabria) (González Sainz y San Miguel 2001).

Tal y como apuntó D. Garate (2010), aunque a veces estas grafías están en zonas cercanas a la boca de entrada (La Pasiega C, Mazaculos, La Garma), lo habitual es su distribución por zonas medias y terminales de la cueva, pudiendo situarse en divertículos laterales (La Pasiega A, El Castillo) (Fig. 7). Estos

\footnotetext{
2 Observado en una visita de Olivia Rivero y Sergio Salazar a la cueva el 20 de enero de 2017.

3 Se trataría de las cuevas de Entrecueves, Tito Bustillo, El Buxu, Mazaculos I, Las Herrerías, Llonín, Las Aguas, Altamira, Chimeneas, La Pasiega A, La Pasiega B, La Pasiega C y La Pasiega D, La Garma, La Llosa, Cofresnedo, Solviejo, Covalanas, Arco B y Arenaza. (Cartailhac y Breuil 1906; Breuil y Obermaier 1935; Berenguer 1979; Fortea, 1981; Moure et al. 1990; Gómez et al. 1991; Cacho et al. 2000; González Sainz y San Miguel 2001; Smith 2002; Balbín et al. 2003; González Sainz 2003; Garate 2004; González Sainz y Balbín 2010; Menéndez 2016; Montes Barquín et al. 2016, 2017; Martínez-Villa 2018)
} 


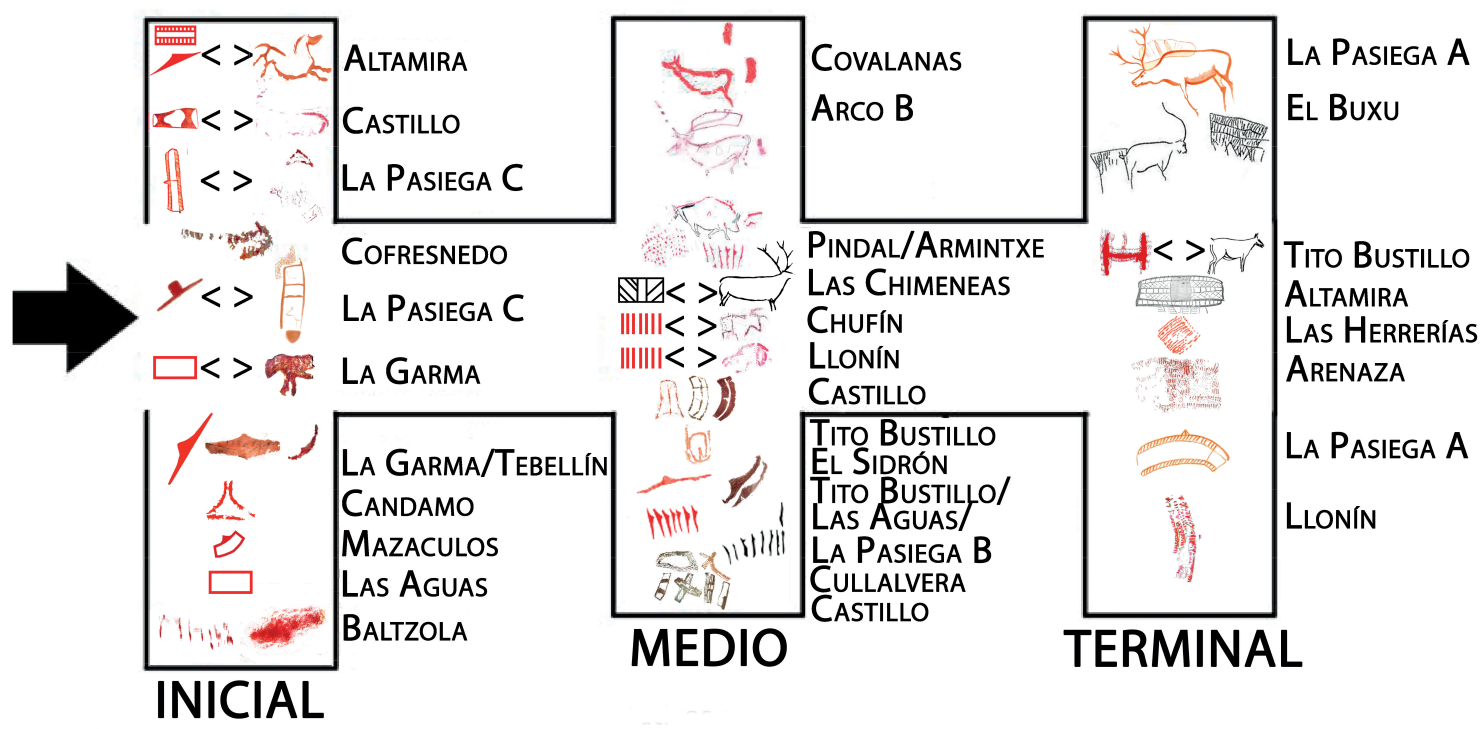

Fig. 7. Distribución de los signos más representativos de la Cornisa Cantábrica desde la entrada al interior de las cavidades (autor D. Garate, en color en la edición electrónica).

signos acolados suelen representarse con divisiones internas (tripartitas, escaleriformes, etc.) (Sauvet et al. 2018), o sin relleno, salvo en La Garma que cuenta con los únicos ejemplos con relleno interior rojo (González Sainz 2003). Además, están en un sector muy próximo a la entrada (sector Ig), compartiendo el mismo lienzo con un claviforme cantábrico.

Sin embargo, la rigidez de las tipologías presentadas para estos ideomorfos pudiera obviar la existencia de límites difusos entre ellas. Ciñéndonos a la morfología de las grafías, podríamos clasificar a los signos "claviformes cantábricos" como "cuadriláteros con apéndice y relleno interior" (al menos en los de base ancha) o decir que ciertos signos cuadriláteros presentan en su interior negativos de claviforme, como en un caso pintado en la cueva del Castillo (Fig. 8).

Podemos limitarnos a los signos cuadriláteros con apéndice y excluir de este grupo el resto de signos cuadrangulares hallados en la Cornisa Cantábrica, incluidos los cuadriláteros negros de Altamira, con una datación C14-AMS de $15.440 \pm 200$ años BP y los de Las Chimeneas (con una datación en el mismo

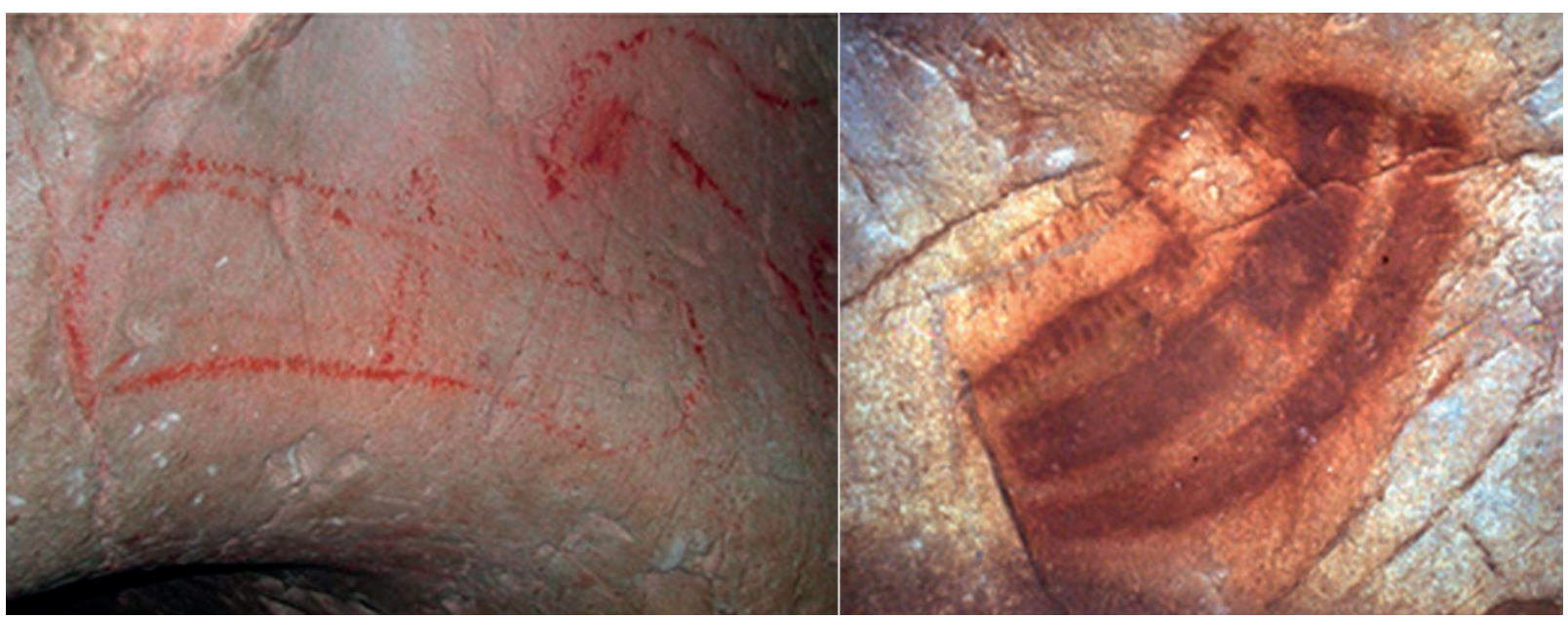

Fig. 8. A la izquierda signo cuadrilátero con apéndice de la cueva de La Pasiega A (autor D. Garate) y a la derecha signo cuadrilátero de la cueva de Castillo, con un negativo de claviforme en su interior (selección de Mingo Álvarez 2010: 75, fotografía 1PS.18). 
panel de $13.940 \pm 140$ años BP) (Moure et al. 1996). En ese caso, estas manifestaciones gráficas, pese a su heterogeneidad formal y en menor medida técnica, se asocian mayoritariamente a figuras animales punteadas o de trazo lineal rojas (en La Pasiega A, C y D, El Castillo, La Garma y Arco B). Es decir, estaríamos ante motivos anteriores con casi total seguridad al Magdaleniense.

\section{CONCLUSIÓN}

El descubrimiento del arte parietal paleolítico de la cueva de Baltzola se enmarca dentro de la reactivación de los estudios de arte parietal de los últimos 15 años en todo el oriente de la Cornisa Cantábrica (Garate 2018), con nuevas localizaciones y estudios actualizados de sitios conocidos de antiguo. El que presentamos sobre la cueva de Baltzola ejemplifica claramente la trascendencia de los resultados obtenidos. Es el primer yacimiento arqueológico del que se tiene constancia en Bizkaia y el conjunto más meridional del oriente de la Cornisa Cantábrica que alberga este tipo de signos complejos, hasta ahora desconocidos en el área.

El deficiente estado de conservación que presenta el conjunto de Baltzola (tan solo se han reconocido 2 grafías paleolíticas pintadas en rojo) no ha impedido la identificación, al menos, de un ideomorfo complejo de tradición cantábrica, pero sí su caracterización precisa. Pudiera tratarse de dos "claviformes cantábricos" yuxtapuestos y contrapuestos, o de una figura "cuadrilátera roja con doble apéndice". Sería siempre un signo hasta ahora inédito en la zona oriental de la Cornisa Cantábrica, que permite relacionar este nuevo conjunto parietal con los de Altamira, La Pasiega, Tebellín o La Garma en el centro-oeste de la Cornisa Cantábrica.

La interpretación del motivo como dos "claviformes cantábricos" comprometería la atribución cronológica de estos ideomorfos, ya que, a lo largo de la historia de la investigación, diversos autores los han adscrito a diferentes contextos temporales. En nuestro estudio hemos reconocido un total de 5 morfotipos diferentes en el repertorio conocido, repartidos en 10 conjuntos diferentes de la Cornisa Cantábrica. En 7 de las 9 cuevas en las que se documentan estos signos abstractos (excluyendo del listado a Baltzola), se encuentran en conjuntos decorados con motivos cuadrangulares y figuras animales premagdalenienses rojas de trazo ancho/punteado. A este dato se tendría que sumar la datación ante quem obtenida en el techo de Altami$\mathrm{ra}$, atribuyéndoles una antigüedad al menos de 36.160 \pm 610 años BP. Sin embargo, son mayoritariamente conjuntos de tradición larga, por lo que es muy complicado relacionar directamente este tipo de figuras con una tradición gráfica concreta. Esta categorización cronológica se complicaría todavía más si tenemos en cuenta la probable superposición de al menos un claviforme cantábrico a una figura polícroma de Altamira, datada en el Magdaleniense Medio. En consecuencia no hay hasta la fecha argumentos discriminantes para decantarse por una $\mathrm{u}$ otra datación.

La clasificación del signo como "cuadrilátero con apéndice" quizás permitiera precisar algo más la asignación cronológica por la asociación mayoritaria de los mismos a figuras animales rojas, previas al Magdaleniense Inferior cantábrico. En el contexto geográfico cercano, el conjunto de la cueva de Askondo -con motivos animales atribuidos al Gravetiense- sería el más próximo a Baltzola. Ambas grutas se encuentran en los cursos altos de cuencas fluviales afluentes del río Ibaizabal, próximas a la divisoria de aguas cantábrico/mediterráneas, una zona por tanto de gran interés estratégico, donde la presencia humana está atestiguada al menos desde momentos pertenecientes al Paleolítico Inferior.

\section{AGRADECIMIENTOS}

Los autores quieren agradecer especialmente al Grupo Espeleológico de Matiena (GEMA), por su colaboración en las investigaciones realizadas en la cueva de Baltzola, así como a los revisores externos del manuscrito que ayudaron a mejorar este texto.

\section{BIBLIOGRAFÍA}

Balbín, R. de 2014: "Los caminos más antiguos de la imagen: el Sella". En M. A. de Blas (ed.): Expresión simbólica y territorial: los cursos fluviales y el arte paleolítico en Asturias. Un siglo después del reconocimiento científico de la Cueva de la Peña, Candamo. Real Instituto de Estudios Asturianos. Oviedo: 65-91.

Balbín, R. de; Alcolea, J. J. y González, M. A. 2003: “El macizo de Ardines, Ribadesella, España: un lugar mayor del arte paleolítico europeo". En R. Balbín y P. Bueno (eds.): El Arte prehistórico desde los inicios del siglo XXI. Primer symposium internacional del arte prehistórico de Ribadesella (Ribadesella 2002). Asociación Cultural Amigos de Ribadesella: 91-152. Oviedo.

Balbín, R. de y González-Sainz, C. 1994: "Un nuevo conjunto de representaciones en el sector D2 de la cueva de La Pasiega (Puente Viesgo, Cantabria)". Homenaje a J. González-Echegaray. Altamira 17: 269-280.

Balbín, R. de y González-Sainz, C. 1996: "Las pinturas y grabados paleolíticos del corredor B.7 de la cueva de La Pasiega (Cantabria)". En A. Moure (ed.): El Hombre Fósil 80 años después. Universidad de Cantabria, Fundación Marcelino Botín, Institute for Prehistoric Research. Santander: 271-294.

Barandiarán, J. M. 1932: "Una visita a la cueva de Balzola". Anuario de Eusko Folklore 12: 111-114.

Barandiarán, J. M. 1950: "Bolinkoba y otros yacimientos prehistóricos de la Sierra de Amboto". Cuadernos de Historia Primitiva 2: 75-112.

Barandiarán, J. M. 1961: "Excavaciones en Silibranka, Atxurra y Goicolau”. Vizcaya XVII: 199-219.

Barandiarán, J. M. 1964: “Arqueología en Vizcaya. Excavaciones en Atxuri”. Noticiario Arqueológico Hispánico 6: 24-51. 
Barandiarán, J. M. 1980: “Excavaciones en Axlor. 1967- 1974”. En J. M. Barandiarán Ayerbe (ed.): Obras completas XVII, La Gran Enciclopedia Vasca. Bilbao: 127-384.

Begouen, R.; Fritz, C.; Tosello, G.; Clottes, J.; Pastoors, A. y Faist, F. 2009: Le sanctuaire secret des bisons: Il y a 14000 ans, dans la caverne du Tuc d'Audoubert. Somogy. Paris.

Berenguer, M. 1979: El Arte Parietal Prehistórico de la «Cueva de Llonín» (Peñamellera Alta, Asturias). Instituto de Estudios Asturianos, Oviedo.

Berganza Gochi, E.; Muñoz Salvatierra, M.; Marcos Muñoz, J. L. 1979: "Excavación de los enterramientos del locus I de la cueva de Balzola (Dima, Vizcaya)". Munibe 31: 189-194.

Breuil, H. y Obermaier, H. 1935: La Cueva de Altamira. Academia de la Historia. Madrid.

Cacho, R.; Muñoz, E.; Montes, R. y González Sainz, C. 2000: “Documentación del yacimiento y las manifestaciones rupestres paleolíticas de la cueva de La Llosa en Obregón, Villaescusa”. En Actuaciones arqueológicas en Cantabria 1984-1999. Consejería de Cultura, Turismo y Deporte, Gobierno de Cantabria, Santander: 305-306.

Cartailhac, E. y Breuil, H. 1906: La Caverne d'Altamira à Santillana près Santander (Espagne). Imprimerie de Monaco. Monaco.

Díaz, Y. y Astorqui, Á. 2012: "Nuevos hallazgos de arte parietal y mobiliar en la cueva de La Cullalvera (Ramales de la Victoria, Cantabria)". Zephyrus 69: 191-198.

Fernández García de Diego, F. 1971: "Aportación al descubrimiento de nuevas figuras parietales en el País Vasco". Munibe 23: 399-404.

Fortea, J. 1981: "Investigaciones en la cuenca media del Nalón Asturias (España) Noticia y primeros resultados". Zephyrvs 32: 5-16.

Fortea, J. 2005: "La plus ancienne production artistique du paléolithique ibérique". En A. Broglio y G. Dalmeri (eds.): Pitture paleolitiche nelle prealpi venete. Grotta di Fumane e Riparo Dalmeri. Memorie del Museo Civico di Storia Naturale di Verona. Preistoria Alpina nr. speciale 9: 89-99.

Gálvez Cañero, A. 1912: Nota acerca de las cavernas de Vizcaya. Boletín del Instituto Geológico y Minero 33, Madrid.

Garate, D. 2004: "Nuevas investigaciones sobre el arte paleolítico de la cueva de Arenzana (Galdames, Bizkaia)". Munibe AntropologiaArkeologia 56, 3-17.

Garate, D. 2010: Las ciervas punteadas en las cuevas del Paleolítico. Una expresión pictórica propia de la cornisa cantábrica. Munibe (Antropologia- Arkeologia), supl. 33, Donostia- San Sebastián.

Garate, D. 2018: "New insights into the study of Paleolithic Rock Art: dismantling the Basque Country void". Journal of Anthropological Research 74 (2): 168-200. https://doi.org/10.1086/695721

Garate, D. y Ríos-Garaizar, J. 2011: "La grotte d'Askondo: un nouveau site ornée dans le golfe de Gascogne". International Newsletter on Rock Art 6: 3-9.

Garate, D. y Ríos-Garaizar, J. 2012: La cueva de Askondo (Mañaria, Vizcaya): arte parietal y ocupación humana durante la Prehistoria. Kobie (serie BAI) 2, Diputación Foral de Bizkaia. Bilbao.

Garate, D. y Ríos-Garaizar, J. 2013: "El arte parietal paleolítico de la cueva de Askondo (Mañaria, Vizcaya)". En C. de las Heras, A. Lasheras, A. Arrizabalaga y M. de la Rasilla (eds.): Pensando el Gravetiense: nuevos datos para la región cantábrica en su contexto peninsular y pirenaico. Monografías del Museo Nacional y Centro de Investigación de Altamira 23, Ministerio de Educación y Cultura, Madrid: 512-525.

García-Díez, M. 2015: "Grafismos rupestres grabados en la cueva de Bolinkoba". En M. J. Iriarte-Chiapusso y A. Arrizabalaga (eds.): Bolinkoba (Abadiño) y su yacimiento arqueológico: Arqueología de la Arqueología para la puesta en valor de su depósito, a la luz de las excavaciones antiguas y recientes. Kobie (serie BAI) 6. Diputación Foral de Bizkaia. Bilbao: 165-172.

GEMA 1999: El otro parque de Urkiola. Departamento de Agricultura de la Diputación Foral de Bizkaia, Bilbao.

Gómez, J.; Malpelo, B.; Serna, A.; Smith, P. y Muñoz, E. 1991: "Notas acerca de la caverna de Mazaculos I". Arquenas 1: 89-141.

González Morales, M. 1982: "La cueva del Tebellín (Bricia, Llanes, Asturias) y sus pinturas rupestres". Ars Prehistorica I: 169-174.

González Sainz, C. 1993: "En torno a los paralelos entre el arte mobiliar y el rupestre". Veleia 10: 39-56.
González Sainz, C. 2003: "El conjunto parietal paleolítico de la galería inferior de la Garma (Cantabria). Avance de su organización interna”. En P. Bueno y R. de Balbín (eds.): El Arte prehistórico desde los inicios del siglo XXI. Primer symposium internacional del arte prehistórico de Ribadesella (Ribadesella 2002): 201-222. Ribadesella.

González Sainz, C. 2012: "Una introducción al arte parietal paleolítico en la Región Cantábrica". En Arte sin artistas. Una mirada al Paleolítico. Catálogo de la exposición Museo Arqueológico Regional. Alcalá de Henares: 152-183.

González Sainz, C. 2017: "Sobre la organización de las decoraciones parietales paleolíticas. Impresiones a partir de Santimamiñe, Armintxe y otros conjuntos rupestres de Bizkaia". En D. Garate y M. Unzueta (eds.): Redescubriendo el arte parietal paleolítico. Últimas novedades sobre los métodos y las técnicas de investigación (Bilbao 2016), Kobie (serie Anejos) 16, Diputación Foral de Bizkaia, Bilbao: 135-147.

González-Sainz, C. y Balbín, R. de 2010: “La Pasiega”. En Asociación Cántabra para la Defensa del Patrimonio Subterráneo (eds.): Las cuevas con arte paleolitico en Cantabria. ACDPS y Cantabria en Imagen. Santander: 191-204.

González Sainz, C. y González Morales, M. 1986: La prehistoria en Cantabria 1. Ediciones Tantin, Santander.

González Sainz, C.; Muñoz, E. y Morlote, J. M. 1997: “De nuevo en la Cullalvera (Ramales, Cantabria). Una revisión de su conjunto rupestre paleolítico". Veleia 14: 73-10.

González Sainz, C. y San Miguel, C. 2001: Las cuevas del desfiladero: Arte rupestre paleolitico en el valle del rio Carranza (CantabriaVizcaya). Ed. Universidad de Cantabria, Santander.

Le Quellec, J. L.; Duquesnoy, F. y Defrasne, C. 2015: “Digital image enhancement with DStretch $\AA$ : is complexity always necessary for efficiency?". Digital Applications in Archaeology and Cultural Heritage 2 (2-3): 55-67. https://doi.org/10.1016/j.daach.2015.01.003

Leroi-Gourhan, A. 1968: La Prehistoria del Arte Occidental. Mazenod. Paris.

Leroi-Gourhan, A. 1981: "Les signes parietaux comme 'marqueurs' ethniques”. En Altamira Symposium. Symposium internacional sobre arte prehistórico (Madrid 1979): 289-294. Madrid.

Lorblanchet, M.; Delpech, F.; Renault, P. y Andrieux, C. 1973: "La grotte de Sainte-Eulalie à Espagnac (Lot)". Gallia préhistoire 16 (2): 233-325. https://doi.org/10.3406/galip.1973.1436

Martínez-Villa, A. 2017: "Revisión del conjunto de arte paleolítico de la cueva de L'Tebellín en el macizo de la Llera (Posada de Llanes, Asturias, España). Una reflexión sobre el concepto y cronología de los ideomorfos tipo 'claviforme'". Cuadernos de Arte Prehistórico 4: $78-114$

Martínez-Villa, A. 2018: "Cueva de Herrerías (La Pereda, Llanes, España). Descripción de sus manifestaciones de arte parietal y nuevos ideomorfos". En Excavaciones arqueológicas en Asturias 2013-2016. Consejería de Educación y Cultura, Gobierno del Principado de Asturias, Oviedo: 63-80.

Menéndez, M. 2014: "Desde Candamo hasta la cueva del Pindal: un siglo de estudios de arte paleolítico en Asturias". Entamu 18: 205246.

Menéndez, M. 2016: “El arte rupestre”. En M. Menéndez (dir), J. Jordá, J. M. Quesada, J. Rojo y P. Saura: La cueva del Buxu (Cangas de Onís, Asturias) en el centenario de su descubrimiento. Excavaciones Arqueológicas en Asturias. Monografías. IV. Consejería de Educación y Cultura, Gobierno del Principado de Asturias, Oviedo: 65-109.

Mingo Álvarez, A. 2010: Los signos rupestres del Paleolítico: la cueva de El Castillo (Puente Viesgo, Cantabria). GEA Patrimonio, Guadalajara.

Montes Barquín, R.; Bayarri Cayón, V.; Muñoz Fernández, E.; Marlote Expósito, J. M.; Herrera López, J. y Ontañón Peredo, R. 2017: “Avance al estudio del registro gráfico paleolítico de la Cueva de Solviejo (Voto, Cantabria, España)". Cuadernos de Arte Prehistórico 3: 39-73.

Montes Barquín, R.; Muñoz, E. y Gómez, A. J. 2016: "Arte rupestre paleolítico en los yacimientos estudiados en el proyecto 'Los tiempos de Altamira"'. En J. A. Lasheras (ed.): Proyecto de investigación 'Los tiempos de Altamira'. Actuaciones arqueológicas en las cuevas de Cualventi, El Linar y Las Aguas (Alfoz de Lloredo, Cantabria, España). Ministerio de Educación y Cultura. Madrid: 699-774. 
Mortillet, G. de 1883: Le préhistorique antiquite de l'homme. C. Reinwald. Paris.

Moure, A.; González, M. y González Sainz, C. 1990: "Las pinturas rupestres paleolíticas de la cueva de Covalanas (Ramales de la Victoria, Cantabria)". Trabajos de Prehistoria 47: 9-38. https://doi.org/10.3989/tp.1990.v47.i0.555

Moure, A.; González Sainz, C.; Bernardo de Quirós, F. y Cabrera, V. 1996: "Dataciones absolutas de pigmentos en cuevas cantábricas: Altamira, El Castillo, Chimeneas y Las Monedas". En 'El hombre fósil' 80 años después. Universidad de Cantabria. Fundación Marcelino Botín. Institute for Prehistoric Research, Santander: 295-324.

Pike, A. W. G.; Hoffmann, H. L.; García-Diez, M.; Pettitt, P. B.; Alcolea, J.; Balbín, R. de;... y Zilhão, J. 2012: "U-Series Dating of Paleolithic Art in 11 Caves in Spain". Science 336: 1409-1413. https://doi.org/10.1126/science. 1219957

Raux, P. 1996: "La grotte Mazet (Payrignac, Lot): nouvelles découvertes et marquages culturels". Bulletin de la société préhistorique française 93 (2): 201-203. https://doi.org/10.3406/bspf.1996.10139

Regalado, E.; San Pedro, Z.; Zapata, L.; Gallaga, I.; García, M. y Pérez, A. 2012: "Cueva de Balzola: IV Campaña". Arkeoikuska: Investigación arqueológica 2011: 238-240.

Regalado, E.; San Pedro, Z.; Zapata, L.; Gallaga, I.; Pérez, A.; Argote, N.;... y San Emeterio, A. 2014: "Cueva de Baltzola". Arkeoikuska: Investigación arqueológica 2013: 196-199.

Ríos-Garaizar, J.; Garate, D.; Gómez- Olivencia, A. e Iriarte-Avilés, E. 2013: "Investigaciones arqueológicas en la Cueva de Arlanpe (Lemoa, Bizkaia)". En J. Rios-Garaizar, D. Garate y A. Gómez-Olivencia (eds.): La Cueva de Arlanpe (Lemoa): ocupaciones humanas desde el Paleolítico Medio Antiguo hasta la Prehistoria Reciente. Kobie (Serie BAI) 3, Diputación Foral de Bizkaia. Bilbao: 5-37.

San Pedro, Z.; Regalado, E.; Zapata, L.; García, M.; Gallaga, I. y Pérez, A. 2010: "Baltzola (Dima)". Arkeoikuska: Investigación arqueológica 2009: 224-227.

Sauvet, G. ; Bourrillon, R. ; Garate, D. ; Petrognani, S. ; Rivero, O. Robert, E. y Tosello, G. 2018: "The function of graphic signs in prehistoric societies: The case of Cantabrian quadrilateral signs". Quaternary International, 491: 99-109. https://doi.org/10.1016/j.quaint.2017.01.039

Smith, P. 2002: "Cofresnedo". En M. L. Serna, A. Valle y P. Smith (coords.): Las cuevas con arte paleolitico en Cantabria. Asociación Cántabra para la Defensa del Patrimonio Subterráneo. Santander, 237-240.

Vialou, D. 1986: L'art des grottes en Ariège Magdalénienne. Supplement à Gallia Préhistoire XXII, CNRS. Paris.

Zapata, L.; Gallaga, I.; García, M.; Regalado, E.; Ruiz, M. y San Pedro, Z. 2008: "Baltzola (Dima)". Arkeoikuska: Investigación arqueológica 2007: 223-224.

Zapata, L.; Gallaga, I.; García, M.; Regalado, E.; Ruiz, M. y San Pedro, Z. 2009: "Baltzola (Dima)". Arkeoikuska: Investigación arqueológica 2008: 249-250.

Zapata, L.; Gallaga, I.; García, M.; Regalado, E.; Ruiz, M. y San Pedro, Z. 2011: "Cueva de Balzola: III Campaña". Arkeoikuska: Investigación arqueológica 2010: 220-221.

Zapata, L.; Gallaga, I.; Regalado, E.; Ruiz, M.; San Pedro, Z. y Savanti, F. 2007: "Baltzola (Dima)". Arkeoikuska: Investigación arqueológica 2006: 242-245.

Trab. Prehist., 76, N. ${ }^{\circ}$ 2, julio-diciembre 2019, pp. 323-334, ISSN: 0082-5638

https://doi.org/10.3989/tp.2019.12240 